R. DEMAGH

Département Génie civil UHL-Batna, Algérie

F. EMERIAULT

Grenoble-INP

UJF-Grenoble 1

CNRS UMR 5521, 3SR

Grenoble 38041, France

R. KASTNER

INSA-Lyon, LGCIE 69621 France

\title{
Modélisation 3D
} du creusement de tunnel par tunnelier à front pressurisé
dans les sols surconsolidés

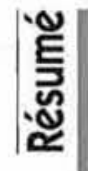

\begin{abstract}
La réalisation de tunnels à faible profondeur en site urbain nécessite une évaluation préalable des effets du creusement sur les ouvrages existants. Dans le cas du creusement par tunnelier à front pressurisé, le phasage des travaux est un problème d'interaction sol/structure hautement tridimensionnel et les différentes opérations réalisées par le tunnelier rendent très complexe une modélisation numérique complète du problème et donc l'évaluation des mouvements induits. Une procédure de simulation tridimensionnelle, utilisant le code en différences finies Flac-3D, prenant en compte de manière explicite les principales sources de mouvements dans le massif encaissant est proposée. Elle est illustrée dans le cas particulier de la ligne B du métro de Toulouse pour lequel des données expérimentales existent, les sols rencontrés étant saturés et fortement surconsolidés $\left(K_{0}\right.$ proche de 1,7$)$. La confrontation des résultats de simulation numérique avec les mesures in situ montre que la procédure de simulation 3D proposée est pertinente, en particulier dans la représentation adoptée pour les différentes opérations réalisées par le tunnelier (excavation, soutènement du front de taille, avancement, pose du revêtement, injection du vide annulaire...).
\end{abstract}

Mots-clés : tunnelier (TBM), simulation numérique 3D, surconsolidation, pression de confinement, injection, mesures in situ.

\section{D modelling of tunnel excavation by pressurized tunnel boring machine in overconsolidated soils}

The excavation of shallow tunnels in urban areas requires a previous evaluation of their effects on the existing constructions. In the case of shield tunnel boring machines, the different achieved operations is a highly three-dimensional problem of soil/structure interaction and are very complex to represent in a complete numerical simulation. Therefore the assessment of the tunnelling-induced soil movements is difficult. A threedimensional simulation procedure, using finite differences code Flac-3D, taking into account in an explicit manner the main sources of movements in the soil mass is proposed. It is illustrated in the particular case of Toulouse subway line B for which experimental data are available and where the soil is saturated and greatly overconsolidated $\left(\mathrm{K}_{0}\right.$ close to 1.7$)$. The comparison of the numerical simulation results with the in situ measurements shows that the $3 \mathrm{D}$ procedure of simulation proposed is relevant, in particular in the adopted representation for the different operations achieved by the tunnel boring machine (excavation, confining pressure, advancement, installation of the tunnel lining, grouting of the annular void...).

Key words: tunnel boring machine (TBM), 3D numerical simulation, overconsolidation, confining pressure, grouting, in situ monitoring. 


\section{Introduction}

Lors du creusement de tunnels par tunnelier à front pressurisé, les déformations et mouvement du terrain environnant ainsi que les tassements de surface sont le résultat d'une succession d'opérations complexes : abattage et soutènement du front de taille, avancement du tunnelier, injection de remplissage du vide annulaire, percolation de coulis et de boue le long du tunnelier, consolidation et prise du coulis..., etc. Cette complexité rend la simulation numérique explicite du creusement difficile. Plusieurs procédures de simulation numérique 3D du creusement de tunnel par bouclier à front pressurisé, dans les sols meubles et aquifères, ont été proposées par divers auteurs durant la décennie écoulée. Parmi les travaux, on citera ceux de Mroueh et Shahrour (1999), Dias et al. (2000), Broere et Brinkgreve (2002), Kasper et Meschke (2004 et 2006) et Bezuijen et al. (2005 et 2006). Les seules références trouvées concernant le creusement dans les sols surconsolidés sont Attewell et Farmer (1974) et Myrianthis (1975). Malgré les progrès actuels, les calculs $3 \mathrm{D}$ restent longs et les problèmes numériques fréquents, ce qui fait que ce type d'approche reste encore peu utilisé. La confrontation à des résultats d'observations, faites sur chantier, montre aussi que les phénomènes induits par le passage d'un tunnelier, provoquant un champ de déplacement tridimensionnel, restent encore mal connus et représentés.

Dans cet article, une procédure de simulation explicite du creusement par phasage d'un tunnel dans un sol surconsolidé avec $K_{0}$ proche de 1,7 est présentée. La procédure est confrontée aux données expérimentales de la section instrumentée Castera, lot 2 de la ligne B du métro de Toulouse, Vanoudheusden et al. (2006). Ces données incluent les mouvements de sol en surface et à l'intérieur du massif, ainsi que les paramètres de conduite du tunnelier. Par ailleurs, une étude paramétrique permet également de valider l'origine du comportement du massif observé et jusqu'à présent non relevé dans la littérature.

\section{Support expérimental}

\section{1}

\section{Présentation de la section de mesures}

Le tunnel du lot 2 est réalisé au moyen d'un tunnelier Herrenknecht à pression de terre de dia mètre $\mathrm{D}=7,7 \mathrm{~m}$, de longueur $\mathrm{L}=8,4 \mathrm{~m}$ et de conicité $\Delta / 2=25 \mathrm{~mm}$ (Fig. 1b). Le profil géotechnique de la section instrumentée Castera est composé d'une molasse essentiellement argileuse, sensiblement homogène, peu perméable et fortement surconsolidée, surmontée d'une couche de remblai. La nappe phréatique se trouve à $4 \mathrm{~m}$ de profondeur, au niveau du toit de la molasse (Fig. 2). Au droit de la section, l'axe du tunnel se trouve à une profondeur $\mathrm{H}=16,5 \mathrm{~m}$ sous une coüverture $\mathrm{C}=12,65 \mathrm{~m}$ (Fig. 1a). Les anneaux, constitués de six voussoirs de $35 \mathrm{~cm}$ d'épaisseur (y compris le voussoir de clé), ont une largeur de 1,40 m. Les paramètres géométriques du tunnel et du bouclier sont résumés dans le tableau I.

L'instrumentation de la section de mesures, qui se situe sur la quatrième inter-station (entre les stations Minimes et canal du Midi), est composée de trois tubes inclinométriques et de 5 extensomètres de forage multipoints avec acquisition automatique (une séquence de mesure complète toutes les 5 secondes). Une description complète de cette instrumentation est donnée sur la figure 2. De plus, lors du passage du tunnelier, un nivellement de précision a été effectué en fin de foration de chaque anneau. En outre, les déformations du revêtement du tunnel ont été mesurées dans l'anneau posé au droit de la section par cinq paires de jauges extensométriques à corde vibrante, situées dans chacun des voussoirs constituant l'anneau (5 voussoirs universels et un voussoir de clé).

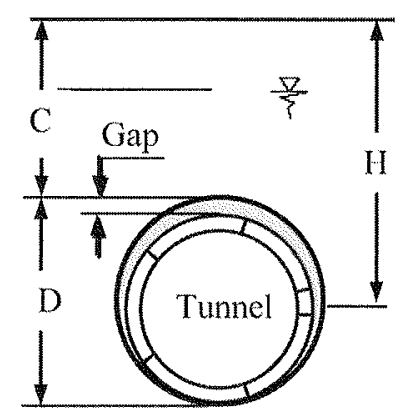

(a)

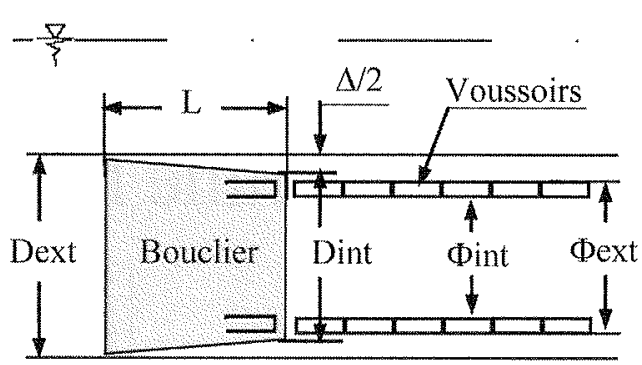

(b)

tic. $f$ Paramètres géométriques du tunnel et du bouclier, (a) section transversale, (b) section longitudinale.

Tunnel and shield geometrical parameters, (a) transverse section, (b) longitudinal section.

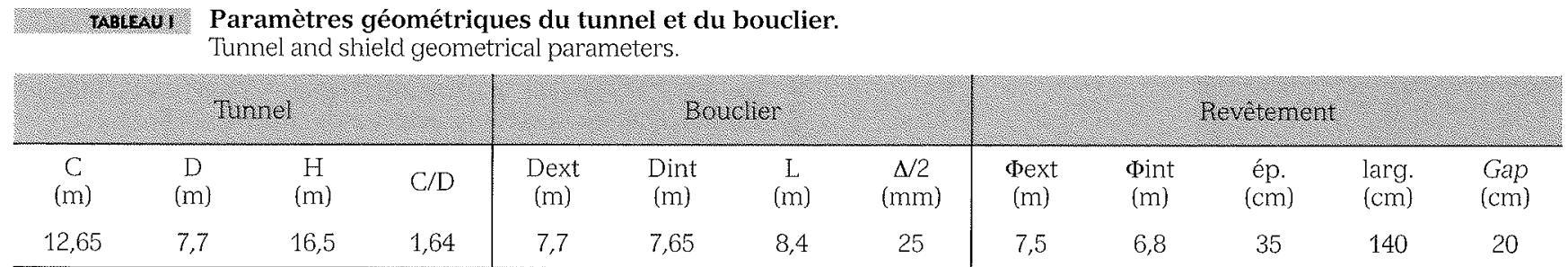



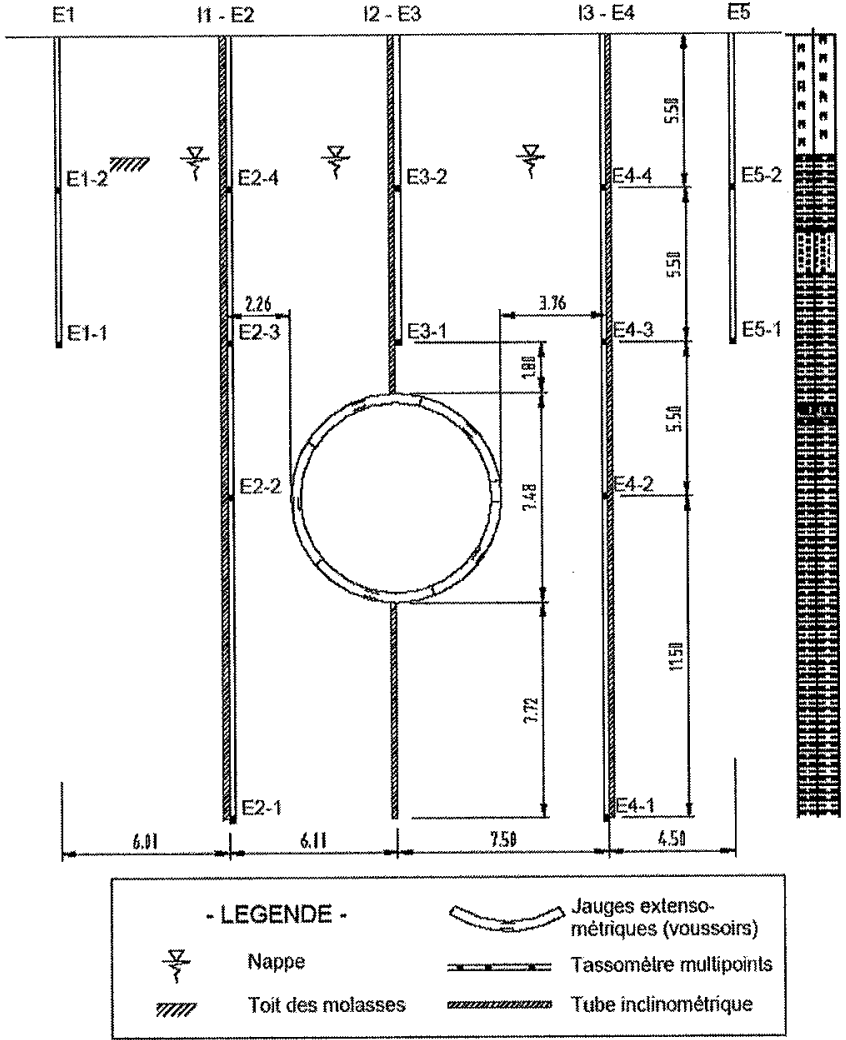

1. 16.2 Section instrumentée Castera.

Castera section.

Les paramètres du remblai et de la molasse argileuse sont résumés dans le tableau II. Le module de déformation de la molasse argileuse est constant et égal à $165 \mathrm{MPa}$ dans les dix premiers mètres, au-delà, il dépend de la profondeur, suivant la relation linéaire $E(z)=9,9 z+66,1$ obtenue par calage sur une autre section de contexte géologique similaire Vanoudheusden et al. (2006).

\section{9}

\section{Paramètres de fonctionnement du tunnelier}

Le tunnelier présente au passage de la section Castera [de -20 à $+40 \mathrm{~m}$ ] une vitesse de progression sensiblement constante et égale à $1,33 \mathrm{~m} /$ heure, ce qui correspond à la pose d'un voussoir toutes les heures (Fig. 3).

Les principaux paramètres de conduite du tunnelier sont reportés sur la figure 4. La figure 4a présente l'évolution de la pression de confinement du front $\mathrm{P}_{\text {front }}$ (rapportée à la contrainte verticale initiale en clé $\sigma_{\mathrm{v0}}^{\mathrm{cle}}$ ), le rapport $\mathrm{P}_{\text {front }} / \sigma_{\mathrm{v0}}^{\text {clé }}$ est pratiquement constant et égal à 0,6 . La figure $4 \mathrm{~b}$ montre l'évolution de la pression

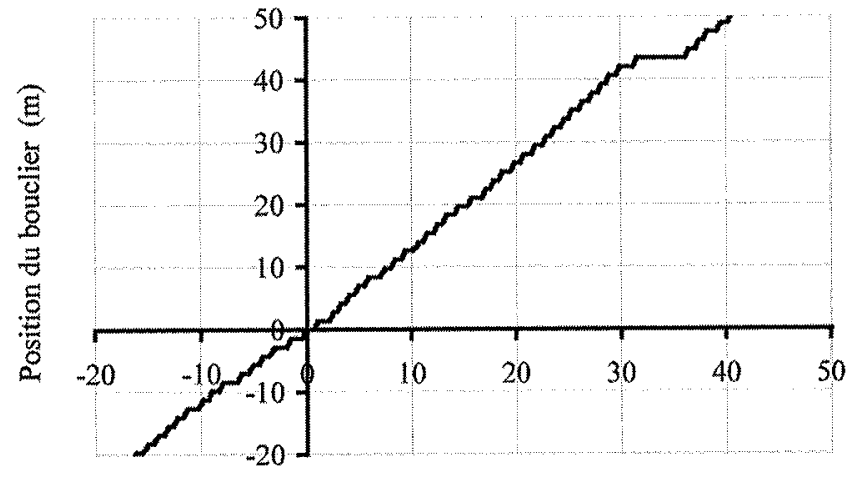

Nombre d'heures depuis le passage (heures)

136.3 Vitesse d'avancement du tunnelier au passage sous la section Castera.

Advancement speed when shield is passing under Castera section.

d'injection du vide annulaire (mesurée à la sortie des quatre pipes d'injection situées en partie supérieure de la jupe du bouclier et rapportée elle aussi à la contrainte verticale initiale en clé $\sigma_{\mathrm{vo}}^{\text {clé }}$. Les rapports

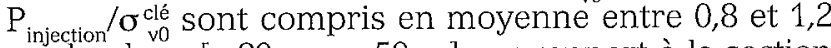
sur la plage $[-20 \mathrm{~m} ;+50 \mathrm{~m}]$ par rapport à la section de mesure. Au passage de la section proprement dite, la pression moyenne d'injection est égale à $0,9 \sigma_{v 0}^{\text {clé }}$ pour connaître ensuite une augmentation qui atteint

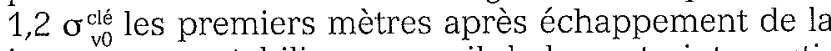
jupe pour se stabiliser au seuil de la contrainte verti-

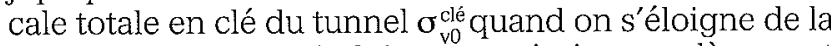
section. Ces valeurs induisent, a priori, un soulèvement millimétrique du sol de surface au moment de l'échappement de la jupe après injection.

Les valeurs enregistrées des principaux paramètres de conduite du tunnelier permettent d'assurer, dans le contexte géologique de l'agglomération toulousaine, une stabilité suffisante du front de taille puisque aucun mouvement de sol majeur n'a été mesuré à l'approche ou lors du passage du bouclier. Compte tenu de cela, cette section constitue un bon exemple de simulation. On retiendra des résultats expérimentaux les points suivants :

- un soulèvement de 1,2 mm observé en surface au droit de l'axe du tunnel;

- une cuvette de soulèvement mise en évidence au passage du tunnelier avec une demi-largeur i égale à $8 \mathrm{~m}$, Peck (1969) ;

- une convergence horizontale au niveau des reins dans la section transversale de 5 à $6 \mathrm{~mm}$.

Ce comportement particulier a été attribué au caractère surconsolidé de la molasse qui présente un coefficient des terres au repos $\mathrm{K}_{0}$ largement supérieur à 1 .

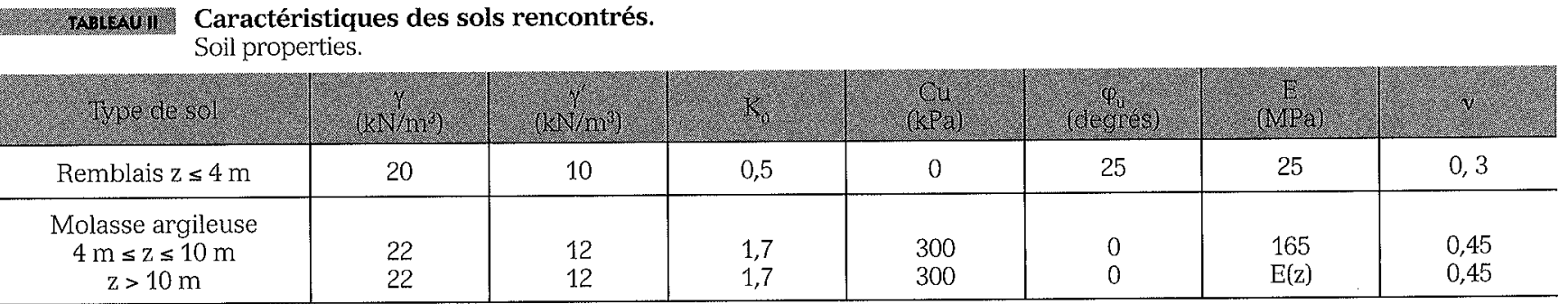



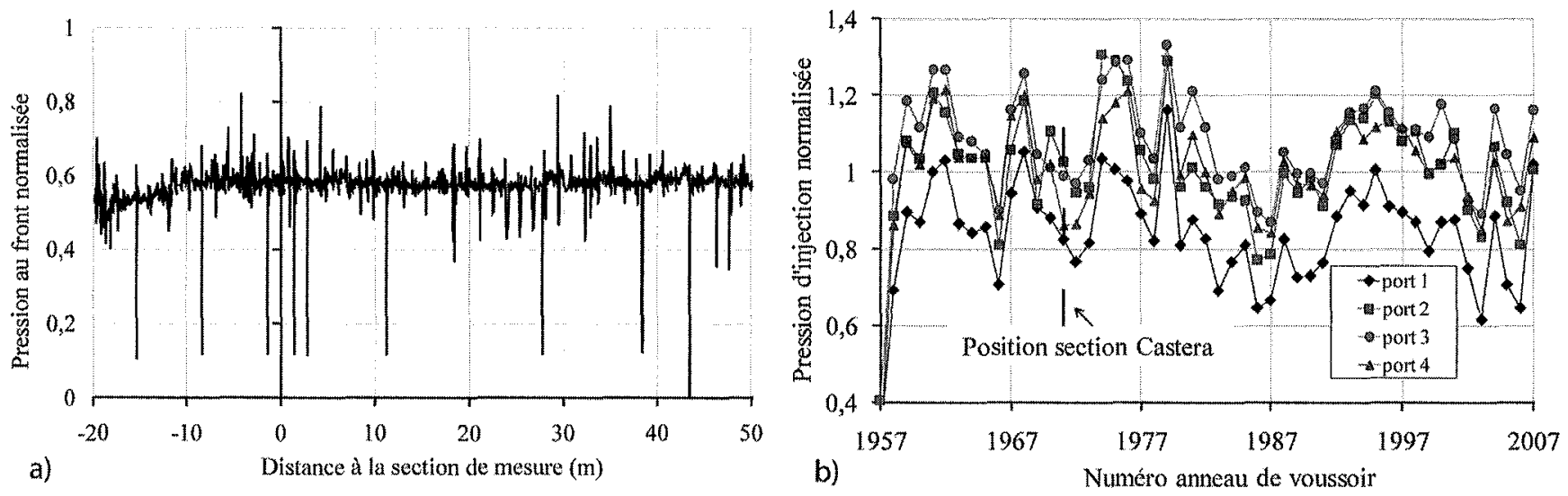

16. 4. Évolution des paramètres de conduite du tunnelier, a) pression au front, b) pression d'injection. Evolution of shield driving parameters, a) front pressure, b) injection pressure.

3

\section{Simulation du creusement}

\section{1}

\section{Procédure de simulation proposée}

La procédure de simulation du creusement proposée essaie de décrire le plus finement possible l'ensemble des opérations réalisées par le tunnelier ainsi que les phénomènes associés (Fig. 5).

Un bouclier de forme conique, parfaitement rigide, les nœuds sont fixés selon la méthode dite de centre fixe, modélisé avec des éléments volumiques minces est installé dans un massif vierge pour lequel un état de contrainte géostatique est instauré avec la méthode $\mathrm{K}_{0}$. Dès lors que le bouclier est totalement mis en place, la procédure de phasage du creusement de la figure 5 peut être appliquée.

L'excavation est simulée par la désactivation d'un disque d'éléments de sol, de longueur fixe égale à la longueur du voussoir. La stabilité du front de taille est contrôlée par la pression normale enregistrée nommée
$\mathrm{P}_{\text {front }}=0,6 \sigma_{\mathrm{v} 0}^{\text {clé }}$ et présentant un gradient de $22 \mathrm{kN} / \mathrm{m}^{3}$ par mètre de profondeur (égal au poids des terres excavées), cette pression de soutènement progresse avec le bouclier. Le passage de ce dernier, simulé par l'annulation des contraintes tangentielles locales, dégage une perte de volume qui est aussitôt comblée par le déconfinement du sol en l'absence d'injection avec la prise en compte de grands déplacements (Fig. 6).

L'interface qui est solidaire du bouclier est activée dès lors qu'un contact est établi avec le sol environnant, le rôle de cette interface étant de bloquer le déconfinement radial du sol mais également de permettre le déconfinement tangentiel par effet de voûte de déformation transversale (Fig. 6b). Le choix d'une interface glissante est justifié par la lubrification probable due à la migration du mortier à l'amont des pipes d'injection (la prise en compte de frottement à l'interface sol/bouclier pilote le comportement différé du sol ce qui va à l'encontre du calcul à court terme mené dans cette étude). Durant la progression du bouclier au sein du massif, la perte de volume est partiellement compensée par la migration possible du mortier vers l'avant du bouclier (il y a une grande incertitude sur la forme de fermeture du sol autour du bouclier). Deux

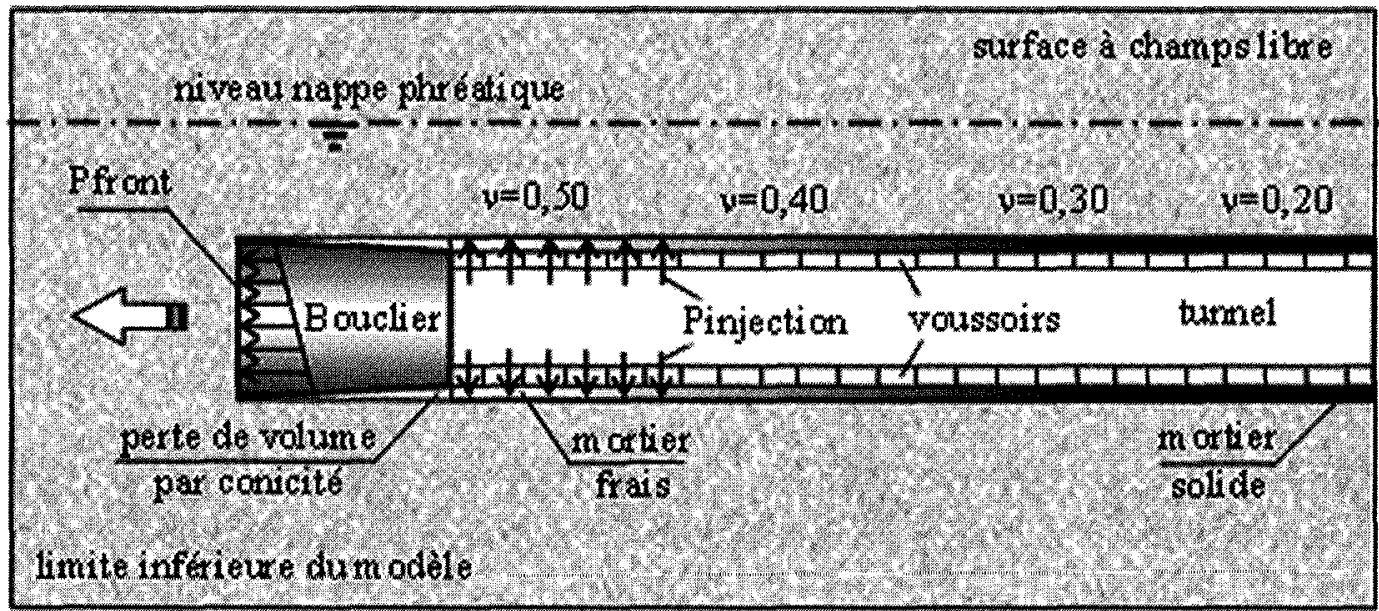


techniques sont utilisées pour simuler cette migration, soit par une pression appliquée sur une certaine longueur arrière du bouclier; soit par une correction de la conicité, calée de manière à reproduire un déplacement enregistré sur chantier. Pour Dias et al. (2000) et Demagh et al. (2008a), cette deuxième technique est plus pertinente.

Le revêtement, simulé par un modèle en long, peut être constitué soit d'éléments coques soit d'éléments volumiques. Il est caractérisé par une rigidité équivalente $\mathrm{E}_{\text {réduit }}=25000 \mathrm{MPa}$ plus faible pour tenir compte des joints d'étanchéité entre les voussoirs préfabriqués, Thépot (1999).

L'injection du coulis dans le vide annulaire (Gap de $20 \mathrm{~cm}$ ) est contrôlée en volume et en pression. Le choix du schéma appliqué de la pression $\mathrm{P}_{\text {injection }}$ sur la figure 7 est justifié par la position des pipes d'injection situées sur la partie supérieure de la jupe du bouclier. Le calage de la pression d'injection est fait sur la mesure d'un déplacement vertical au plus près de la clé du tunnel (extensomètre E3-1 en l'occurrence). Il montre en particulier que la pression réellement transmise au sol reste inférieure à la pression moyenne enregistrée sur les pipes d'injection. Cette différence est due à la perte de charge par frottement suite à l'écoulement du mortier ainsi qu'à son imprégnation du sol environnant. L'incertitude sur la rhéologie du mortier amène à considérer deux phases principales (phases liquide et solide) intercalées par une ou deux phases transitoires (Fig. 6).

La phase liquide qui correspond au comportement incompressible du mortier pour combler le vide annulaire et transmettre la pression d'injection au sol environnant. Cette phase est simulée par l'application d'une pression $\mathrm{P}_{\text {injection }}$ sur la paroi de l'excavation et la réactivation d'eléments volumes. Un gradient de pression est considéré pour tenir compte non seulement du poids propre du mortier mais également des dispositions particulières d'injection. Le mortier est considéré élastique incompressible, Dias et al. (2000), avec initialisation d'un état isotrope de compression égal au schéma de pression appliquée. Durant cette phase, le mortier est caractérisé par un fort module de compressibilité $\mathrm{K}$ associé à un faible module de cisaillement $\mathrm{G}\left(\mathrm{K}=10^{3} \mathrm{MPa}\right.$ et $\left.\mathrm{G}=1 \mathrm{kPa}\right)$, Bezuijen et al. (2006). Cette phase dure aussi longtemps que le mortier garde entière son ouvrabilité, soit environ quatre heures d'après Talmon et al. (2005), ce qui correspond à la pose d'un certain nombre de voussoir.

La phase de transition, qualifiée de consolidation/ prise du mortier, commence quelques mètres après l'injection. Durant cette phase, la rhéologie du mortier évolue plus ou moins rapidement, selon le type de mortier utilisé (actif ou inerte). Plus consistant, le mortier acquiert une résistance au cisaillement associée à une certaine compressibilité acquise au cours de sa consolidation. Cette phase est simulée par l'annulation de la pression d'injection, une augmentation du module de cisaillement $\mathrm{G}$ et une diminution progressive du coefficient de Poisson. Suivant la nature du coulis, cette phase peut durer plus ou moins longtemps.

La phase solide correspond à la situation finale durant laquelle le mortier, plus rigide que le milieu environnant, est en mesure de transmettre les efforts du massif au revêtement Kasper et Meschke (2004). Cette phase est caractérisée par des modules $\mathrm{K}$ et $\mathrm{G}$ à peu près équivalents $(\mathrm{K}=1,33 \mathrm{GPa}, \mathrm{G}=1 \mathrm{GPa})$ Dierkens (2005).

Cette procédure est répétée tout au long de la progression du bouclier, jusqu'à atteindre une section stationnaire après quelques dizaines de passes d'excavation.

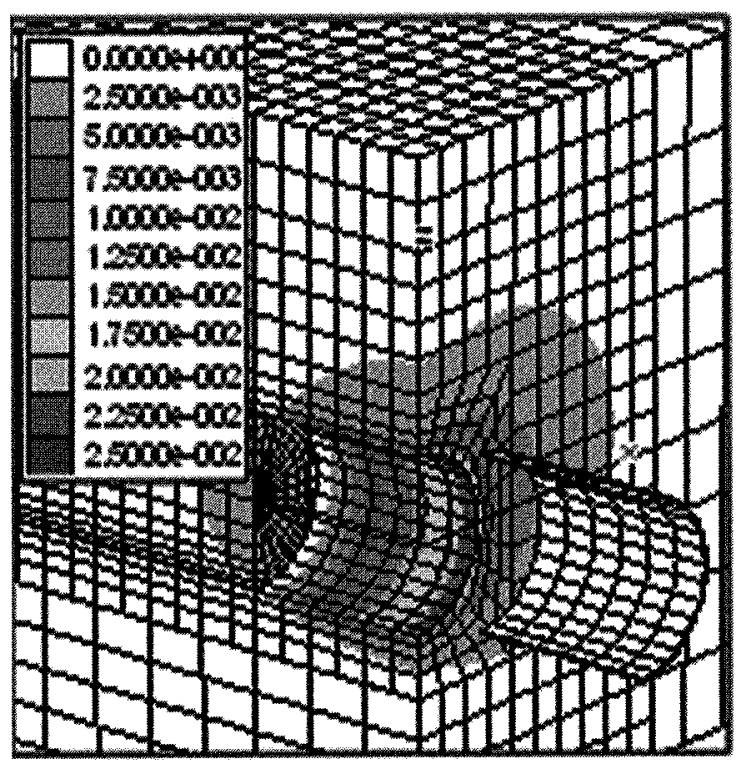

a)

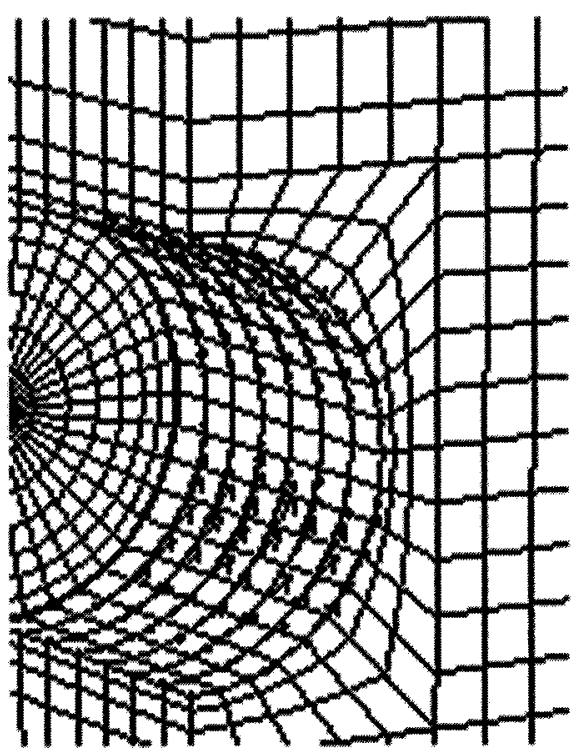

b)

Maillage utilisé, a) contour des déplacements verticaux, b) effet de voûte après installation du bouclier. Mesh used, a) vertical displacements contours, b) arc effect after the installation of the shield. 
Les paramètres ainsi définis sont appliqués au cas de référence. Ils correspondent aux paramètres enregistrés lors du passage de la section de mesure. Étant donné la nature peu perméable des matériaux traversés (la perméabilité étant proche de $10^{-9} \mathrm{~m} / \mathrm{s}$ ), les calculs ont été menés en conditions non drainées (en termes de contraintes totales) avec prise en compte de la nappe phréatique, ce qui correspond au comportement à court terme de la molasse toulousaine. Les paramètres de résistance de la molasse adoptés dans les calculs étant ceux donnés par l'essai triaxial rapide, Vanoudheusden et al. (2006).

Les résultats des simulations $3 \mathrm{D}$ sont confrontés aux mesures enregistrées lors du passage sous la section Castera. Ces données incluent les mouvements de sol en surface et à l'intérieur du massif, ainsi que les paramètres de conduite du tunnelier.

\section{3.9}

\section{Maillage et conditions aux limites}

Le maillage est composé d'éléments brique à huit nœuds avec près de 75000 nœeuds. Les conditions aux limites sont imposées en termes de déplacements nuls dans la direction perpendiculaire aux faces avec prise en compte de la symétrie verticale ce qui permet de limiter la taille du modèle (Fig. 6). L'étendue du maillage dans le sens longitudinal est conditionnée par la position de la section stationnaire après stabilisation des déplacements des points $\Delta \mathrm{V}$ et $\Delta \mathrm{H}$ (Fig. 7), ce qui correspond, pour le cas étudié, à 44 passes d'excavation. Le sol est modélisé en élastoplasticité avec un critère de rupture de type Mohr-Coulomb et une règle d'écoulement non associée nécessitant peu de paramètres. Les dimensions du modèle sont : longueur $=252 \mathrm{~m}$, largeur $=150 \mathrm{~m}$ et hauteur $=30 \mathrm{~m}$.

\section{4}

\section{Analyse des résultats}

\section{1}

\section{Cas de référence}

Qualitativement, les premières simulations sont calées sur les paramètres enregistrés lors du passage sous la section Castera pour analyser le comportement du massif et l'interaction sol/tunnelier avec la progression du creusement. La pression d'injection a été volontairement majorée de $20 \%$ pour mettre en évidence l'effet de l'injection sur celui de $K_{0}$. D'emblée, un fort coefficient de poussée des terres $K_{0}=1,7$ paraissait mieux adapté au contexte géologique particulier de la molasse toulousaine $\left(\mathrm{K}_{0}=1,8\right.$ à $25 \mathrm{~m}$ de profondeur d'après Vanoudheusden et al. (2006)). Le choix du schéma de pression est justifié par la position des pipes d'injection situées sur la partie supérieure de la jupe du bouclier (Fig. 7b). La pression au front est appliquée avec un gradient de profondeur correspond au poids des terres excavées.

Sur la figure 7a, sont représentées les déformations simultanées au sein du massif : le soulèvement vertical $\Delta \mathrm{V}$ du point de surface sur l'axe et à l'aplomb du tunnel (extensomètre $\mathrm{E} 3$ ) et la convergence horizontale $\Delta \mathrm{H}$ du point situé au rein droit du tunnel à une distance de 2,15 $\mathrm{m}$ du bord de l'excavation (inclinomètre I1), en fonction de la position de la tête de foration. On peut distinguer quatre phases :

- $1^{\text {re }}$ phase : l'approche du bouclier, se traduit par une faible convergence au niveau des reins $\Delta \mathrm{H}=1,5 \mathrm{~mm}$, le point $\Delta \mathrm{V}$ étant peu perturbé ;

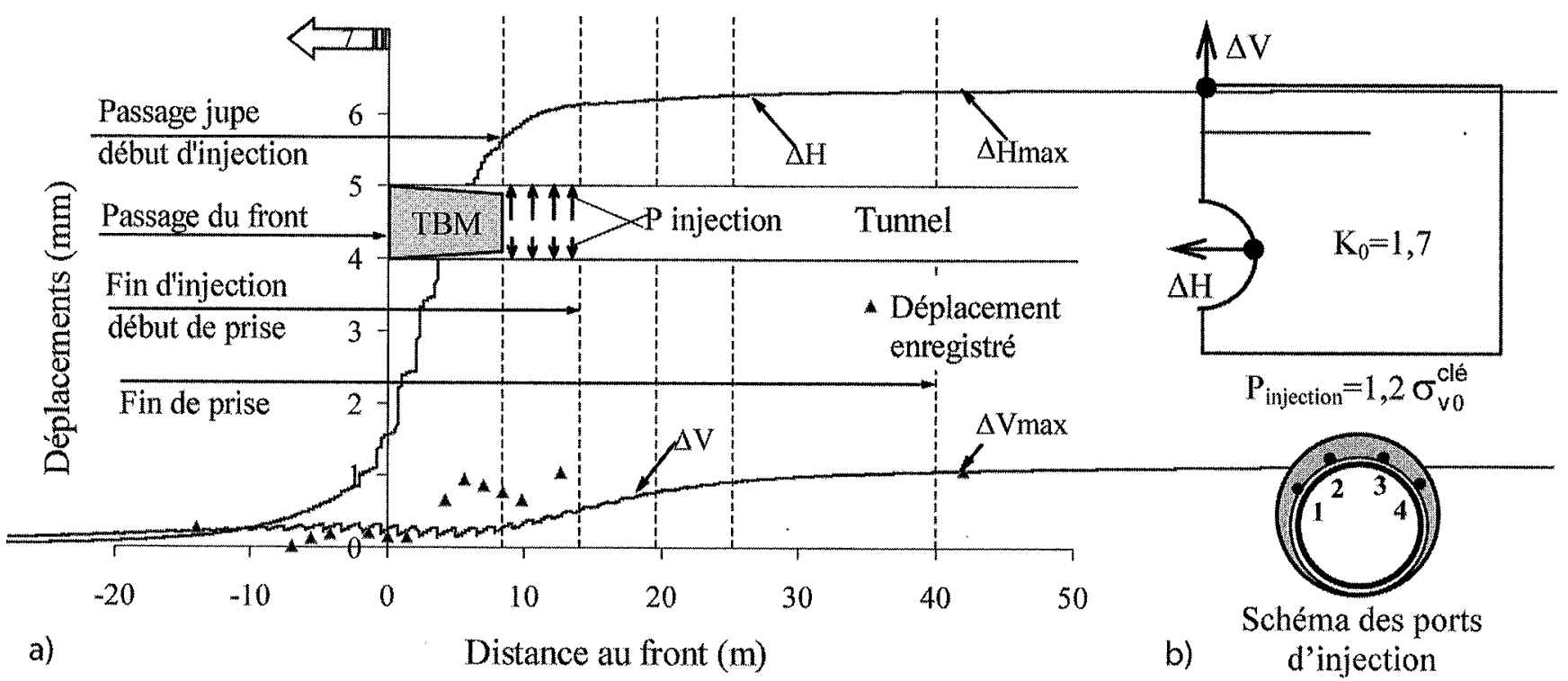


$-2^{e}$ phase $:$ durant le passage de la jupe, $90 \%$ de la convergence totale est atteinte, soit $\Delta \mathrm{H}=5,7 \mathrm{~mm}$. Le déconfinement du sol, à cause de la perte de volume par conicité, se traduit paradoxalement par un faible soulèvement en surface $\Delta \mathrm{V}=0,3 \mathrm{~mm}$;

$-3^{e}$ phase : l'injection du mortier fluide se traduit par un faible soulèvement $\Delta \mathrm{V}=0,6 \mathrm{~mm}$, ce qui met en évidence qu'une partie du soulèvement est aussi due à l'injection (et non pas uniquement par l'effet de $\mathrm{K}_{0}$ ) ;

- $4^{\mathrm{e}}$ phase : à partir de $40 \mathrm{~m}$ par rapport à la section auscultée, la prise/consolidation du mortier se traduit par une stabilisation simultanée de la convergence au niveau des reins à $\Delta H=6,3 \mathrm{~mm}$ et du déplacement vertical à $\Delta V=1,2 \mathrm{~mm}$ et un rapport $\Delta \mathrm{H} / \Delta \mathrm{V}$ voisin de 5 .

Pour la qualification de la procédure de simulation proposée, on a repris les mêmes paramètres à l'exception de la valeur maximale de la pression d'injec-

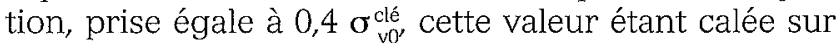
la mesure du déplacement vertical de l'extensomètre situé le plus près de la clé du tunnel (E3-1 en l'occurrence). Ce résultat met particulièrement en évidence l'hypothèse faite a priori que la pression réellement transmise au sol est toujours inférieure à la pression moyenne enregistrée sur les pipes d'injection.

Les comparaisons sont faites sur l'évolution des déformations inclinométriques et de la cuvette transversale de tassement en fonction de la position de la tête de foration par rapport à la section de mesures (Figs. 8 et 9 ).

Qualitativement, ces figures montrent une bonne concordance avec les déplacements mesurés, tant au niveau de leur évolution par rapport à la position de la tête de foration que pour les valeurs finales après

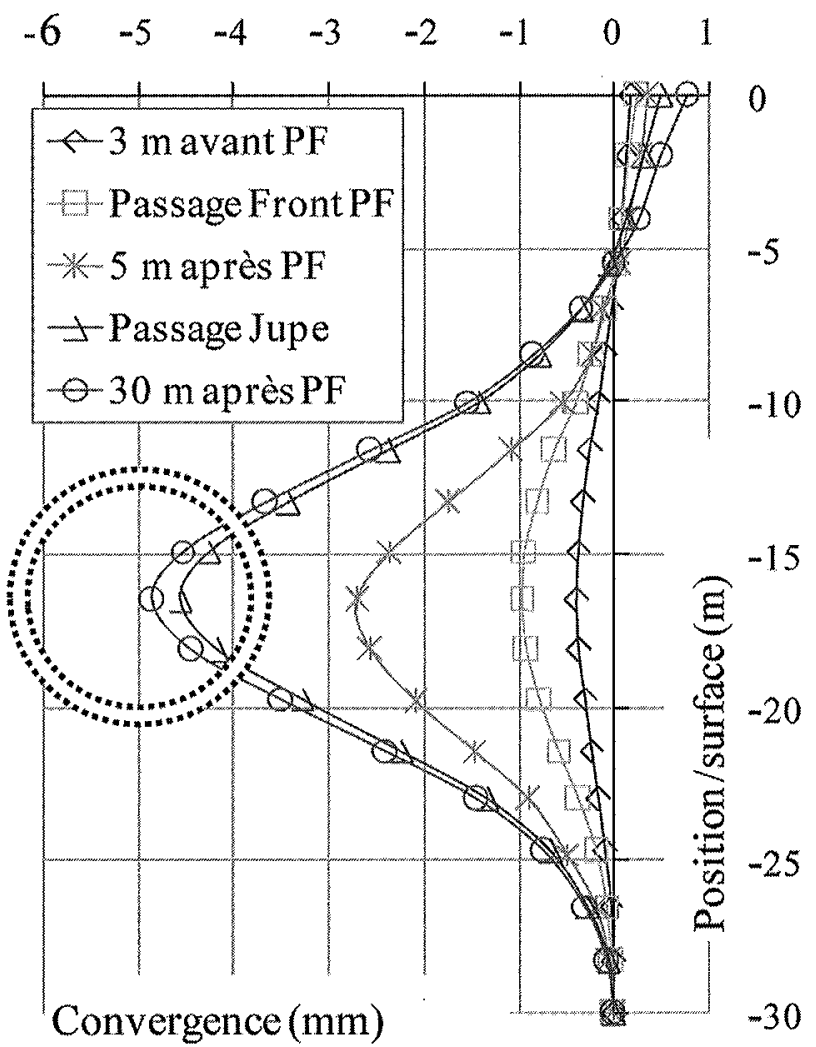

a) stabilisation des déplacements (numériquement, $150 \mathrm{~m}$ après la section de mesure), ce qui, compte tenu d'une vitesse de progression des calculs, égale en moyenne à $1,33 \mathrm{~m} /$ heure, correspond à titre indicatif, à 5 jours de creusement.

En particulier, sur la figure 8a, le choix d'une loi de variation du module de déformation de la molasse plus appropriée que celle proposée au $\$ 2.1$ pourrait affiner encore plus les résultats des déplacements de convergence entre les cotes -5 et $-10 \mathrm{~m}$.

\section{2 \\ Étude paramétrique}

Une étude paramétrique visant à analyser la sensibilité du massif vis-à-vis des paramètres de simulation les plus pertinents a été entreprise (Figs. 10-12). La réponse du massif est analysée à travers les déplacements $\Delta \mathrm{F}, \Delta \mathrm{H}$ et $\Delta \mathrm{V}$, respectivement extrusion axiale du front, déplacement horizontal du point $\mathrm{H}$ et déplacement vertical du point $\mathrm{V}$.

La figure 10a montre une évolution régulière et sensiblement linéaire des déplacements en fonction de $\mathrm{K}_{\gamma}$ ce qui montre, pour une pression d'injection donnée, une sensibilité et un comportement identiques des trois paramètres au coefficient de poussée des terres $\mathrm{K}$. La figure $10 \mathrm{a}$ montre également un soulèvement en surface pour $\mathrm{K}_{0} \geq 1,3$ alors que l'on observe pour des valeurs plus faibles un tassement.

La figure 10b montre la sensibilité des déplacements par rapport au coefficient de Poisson $v$. La pertinence de cette dernière étude est dictée par les temps de calcul très élevés lorsqu'on se rapproche des

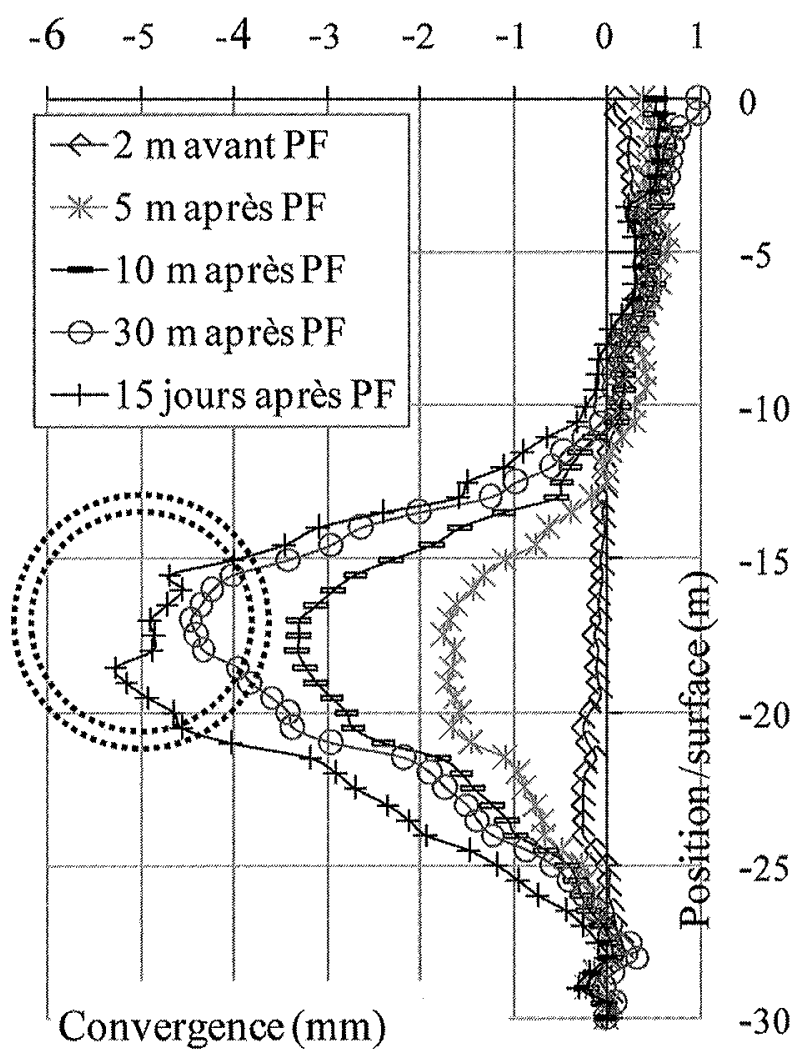

b)

T6. Évolution des déformations inclinométriques, a) simulations Flac-3D, b) mesures enregistrées I3. Horizontal displacement profiles, a) numerical simulation, b) in situ measurements I3. 

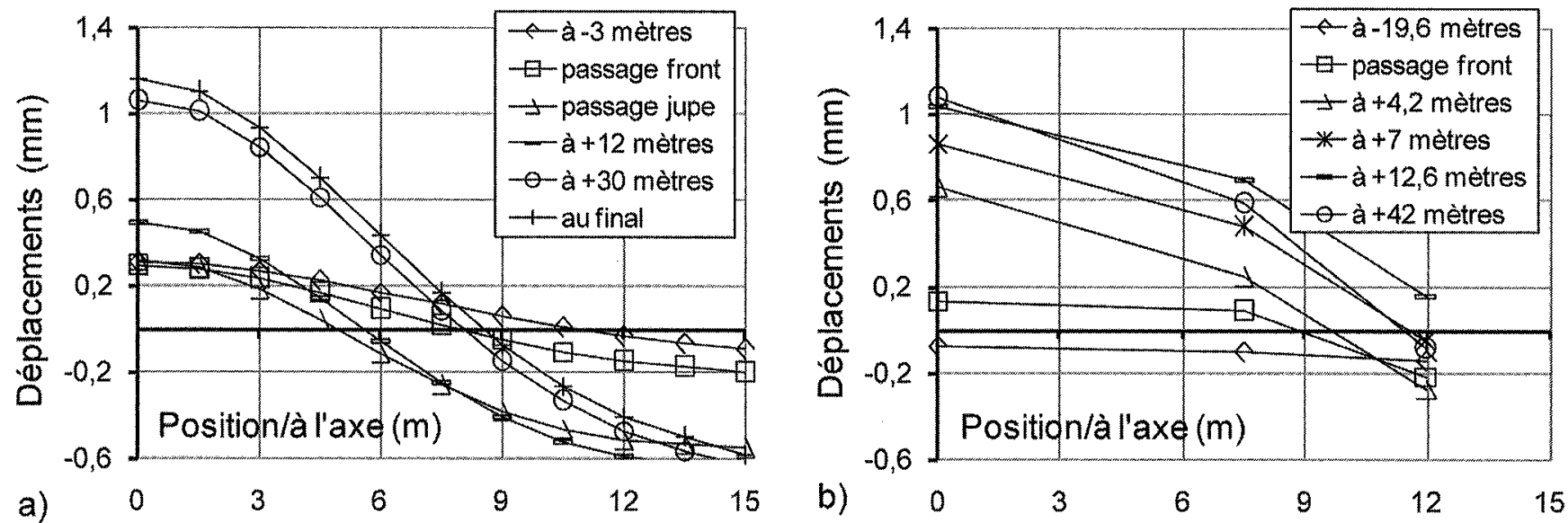

16. 9 Évolution de la cuvette des tassements, a) simulations Flac-3D, b) mesures enregistrées.

Settlement troughs for different positions of the TBM, a) numerical simulations, b) in situ measurements

matériaux parfaitement incompressibles $(v=0,5)$. Elle montre en particulier que les déplacements restent sensiblement les mêmes. Le choix d'un coefficient de Poisson de la molasse égal à $v=0,45$ au lieu de $v=0,49$ diminue par trois les temps de calcul.

Sur la figure 11a, globalement quand la conicité augmente, la convergence $\Delta \mathrm{H}$ augmente et l'extrusion axiale $\Delta \mathrm{F}$ diminue. De même, le soulèvement $\Delta \mathrm{V}$ augmente quand la conicité double de $\Delta / 2=12,5$ à $25 \mathrm{~mm}$ puis $\Delta V$ diminue quand la conicité passe de 25 à $50 \mathrm{~mm}$. Ceci peut être expliqué par le fait que dans le premier cas, le soulèvement est piloté par la convergence $\Delta H$ au niveau des reins, tandis que dans le second cas, le déconfinement au niveau des reins étant total, l'extension verticale de la couverture, Vanoudheusden et al. (2006), atténue le soulèvement et de ce fait $\Delta V$ diminue.

La figure $11 \mathrm{~b}$ montre que l'extrusion $\Delta \mathrm{F}$ diminue quand $\mathrm{P}_{\text {front }}$ augmente, elle montre aussi que la varia-

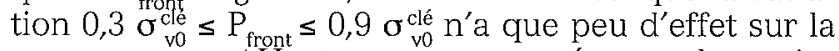
convergence $\Delta \mathrm{H}$ et que par conséquent la variation de $\Delta \mathrm{V}$ reste négligeable. Théoriquement (par la méthode de l'analyse limite), la stabilité du front de taille est assurée, même avec une pression $P_{\text {front }}$ nulle $\left(c_{4}=300 \mathrm{kPa}\right)$, cependant, le calcul en déplacement fait avec pression de soutènement au front $\left(P_{\text {f }}\right.$ $=0$ ) a donné une extrusion axiale rapportée au diamètre de l'excavation de l'ordre de $1 \%$, valeur qui assure l'effondrement du front de taille, Demagh et al. (2008b), ce qui peut être expliqué par l'état de surconsolidation de la mollasse toulousaine $\left(\mathrm{K}_{0}\right.$ proche de $1,7)$. Par contre avec une pression au front $\mathrm{P}_{\text {front }}=1,2$ $\sigma^{\text {clé }}$ on obtient un soulèvement relativement excessif $(\Delta V>2 \mathrm{~mm})$ et qui ne montre aucun signe de stabilisation après 150 passes de calculs.

Sur les trois premiers cas de la figure 12, la pression d'injection augmente avec un même schéma de

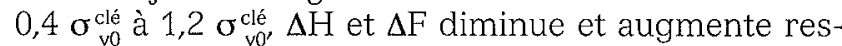
pectivement dans de faibles proportions, tandis que le soulèvement $\Delta V$ augmente de façon significative. Ceci met en évidence l'influence directe de la pression d'injection sur le soulèvement. Les deux derniers calculs montrent particulièrement que le soulèvement reste très sensible au schéma de pression appliqué et donc à la position des pipes d'injection.
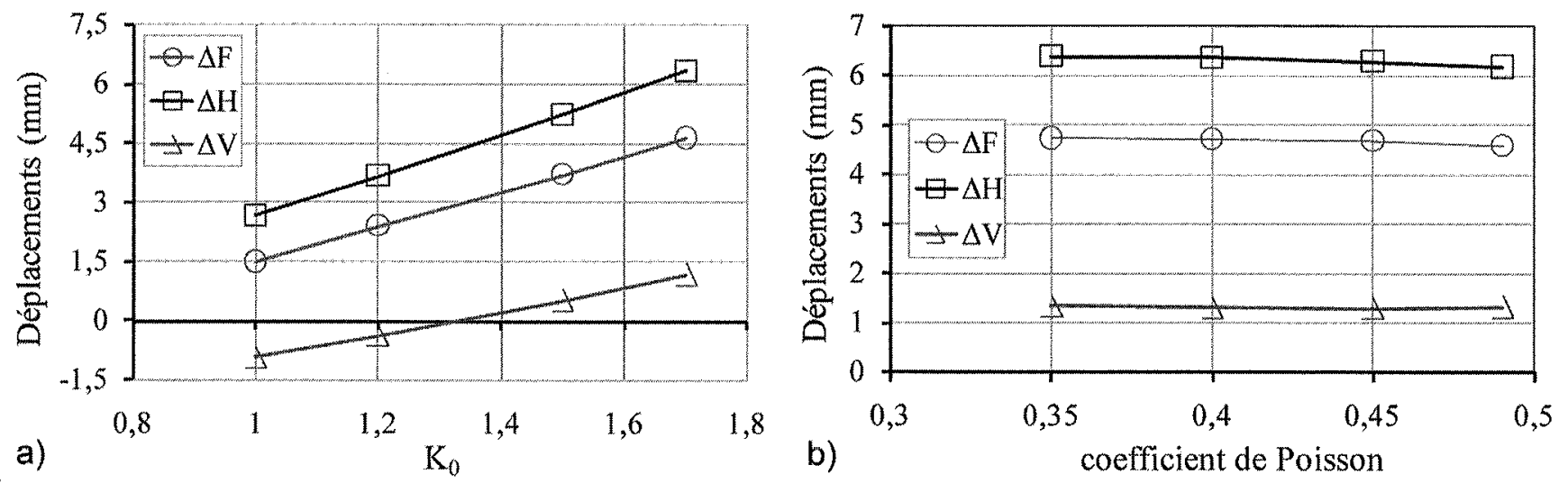


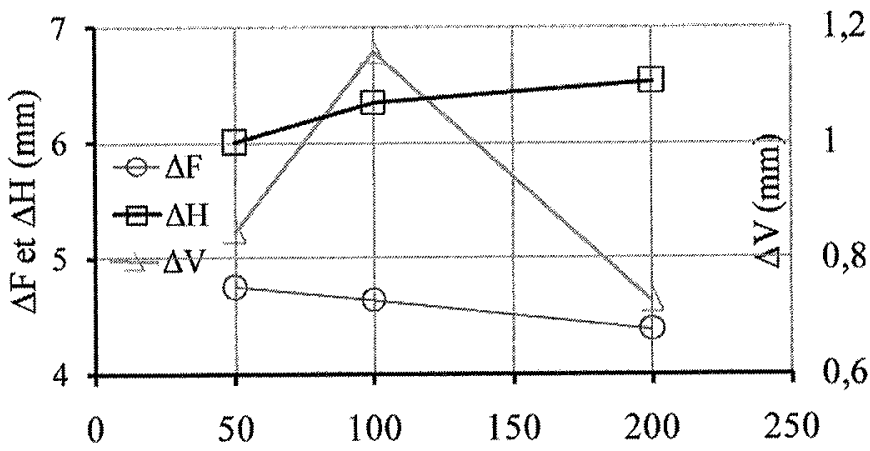

a) Conicité actuelle/conicité réelle (\%)

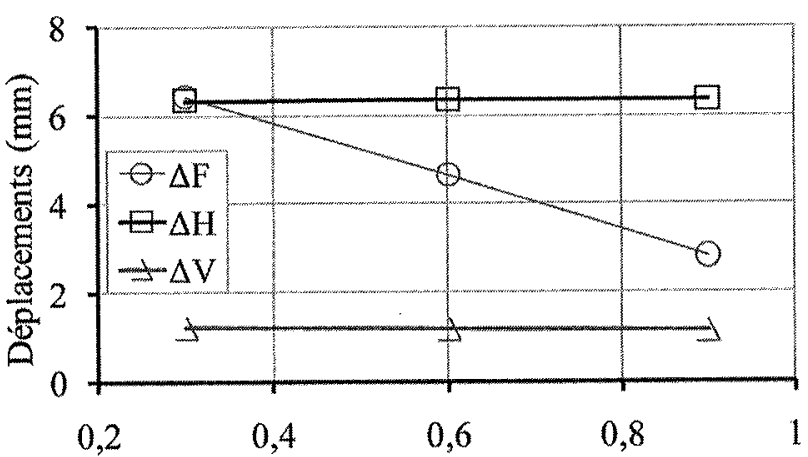

b) Pression confinement/Contrainte verticale

Étude paramétrique de sensibilité, a) conicité, b) pression de confinement.

Parametric study, a) shield conicity, b) face pressure.

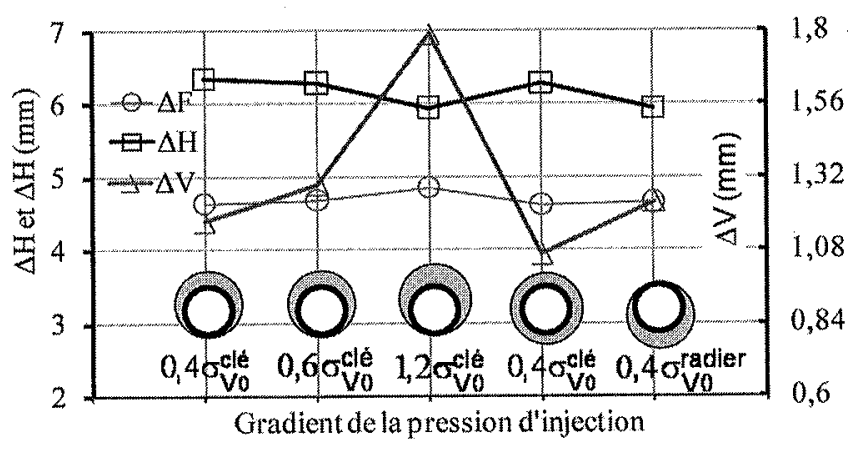

Étude paramétrique de sensibilité à la pression d'injection.

Parametric study of grouting pressure.

\section{Conclusion}

Dans cet article on présente une procédure de simulation tridimensionnelle du creusement de tunnel par tunnelier à front pressurisé. Elle a été appliquée sur un cas réel pour lequel les données expérimentales mettent en évidence un comportement singulier. Les mouvements atypiques de soulèvement du sol associés à une convergence horizontale au niveau des reins ont ainsi pu être attribués en partie à la nature fortement surconsolidée de la molasse toulousaine. Pour le coefficient des terres au repos $K_{0}$, la valeur de 1,7 fournit une bonne concordance entre les déplacements mesurés et ceux évalués numériquement par la procédure proposée.

Qualitativement, les calculs menés en conditions non drainées ont montré une bonne concordance des déplacements évalués numériquement avec ceux mesurés in situ. La confrontation des résultats de simulations (évolutions des cuvettes transversales et des déformations inclinométriques), avec les enregistrements ont montré que la procédure de simulation 3D proposée est pertinente, en particulier dans la représentation des différentes opérations réalisées par le tunnelier (excavation, soutènement du front de taille, avancement et pose du revêtement, injection du vide annulaire).

Néanmoins, les incertitudes liées à l'injection du mortier subsistent; si la migration du mortier paraît être bien simulée par une conicité corrigée, les différentes phases de l'injection restent encore difficiles à simuler et doivent encore faire appel à une rétro-analyse.

Outre l'influence de $\mathrm{K}_{\alpha}$ l'étude paramétrique a permis de cerner l'impact des paramètres de conduite du tunnelier. Une augmentation de la conicité peut changer, par un jeu subtil de déconfinement horizontal et d'extension verticale, l'amplitude des déplacements en surface. L'influence de la pression d'injection, en particulier sur le déplacement vertical, semble être en accord avec la consigne généralement admise dans les chantiers de creusement à savoir $P_{\text {iniection }}=\sigma_{\mathrm{v} 0}^{\text {clé }}$. La pression de confinement n'a que peu d'effet sur les déplacements, du moins en respectant la consigne $0,3 \sigma_{\mathrm{v} 0}^{\text {clé }} \leq P_{\text {front }} \leq \sigma_{\mathrm{v} 0^{\circ}}^{\text {clé }}$ 


\section{Bibliographie}

Attewell P.B., Farmer I.W. - Ground disturbance caused by shield tunnelling in a stiff, overconsolidated clay. Elsevier, Engineering Geology, 8, 1974, p. 361-381.

Bezuijen A., Talmon A.M., Kaalberg F.J. Plugge R. - Field measurements of grout pressure during tunnelling of Sophia Rail Tunnel. Tunneling. GeoDelft, 2005, p. 83-93.

Bezuijen A., Talmon A.M. - Grout properties and their influence on back fill grouting. Geotechnical Aspects of Underground Construction in soft Ground, Bakker et al. (eds) Taylor \& Francis Group, London, 2006, p. 187-193.

Broere W., Brikgreve R.B.J. - Phased simulation of a tunnel boring process in soft soil. NUMGE, Mestat (ed.), Presses de l'ENPC/LCPC, 2002, p. 529-536.

Demagh R., Emeriault F., Kastner R. Modélisation 3D du creusement de tunnel par tunnelier à front pressurisé dans les sols surconsolidés. Journées nationales de géotechnique et de géologie de l'ingénieur, $J N G G^{\prime} 08$, Nantes, 18-20 juin 2008 (a), p. 305-312.

Demagh R., Emeriault F., Benmebarek S - Analyse numérique de la stabilité du front de taille d'un tunnel à faible couverture en milieu frottant. Revue française de géotechnique, 123, 2008 (b), p. 27-35.

Dias D., Kastner R., Maghazi M. - Three dimensional simulation of slurry shield tunneling. Geotech. aspects of underground const. in soft ground, Kusakabe et al. (eds); Balkema, Rotterdam, 2000, p: $351-356$

Dierkens M. - Mesures rhéologiques et modélisation de matériaux en cours de prise. PhD thesis, INSA-Lyon, 2005.

Kasper T., Meschke G. - A 3D finite element simulation model for TBM tunneling in soft ground. Int. J. Num. and Anal. Meth. in Geomech, 28, 2004, p. $1441-1460$

Kasper T., Meschke G. - On the influence of face pressure, grouting pressure and TBM design in soft ground tunnelling. Tunnelling and Underground Space Technology, 21, 2006, p. 160-171.

Mroueh H., Shahrour I. - Modélisation tridimensionnelle du creusement de tunnels en site urbain. Revue française de génie civil, 3, 1999, p. 7-23.
Myrianthis M.L. - Ground disturbance associated with shield tunnelling, in overconsolidated stiff clay. Rock Mechanics, 7, 1975, p. 35-65.

Peck R.B. - Deep excavations and tunnelling in soft ground. Proceedings of the 7th International Conference on Soil Mechanics and Foundation Engineering, state-of-the-art, Mexico City, Mexico, 1969 , p. 225-290.

Talmon A.M., Aanen L., Bezuigen A., van der Zon, W.H. - Grout pressure around a tunnel linning. Tunneling. A Decade of Progress. GeoDelft, 2005, p. 77-82.

Thépot 0 . - Un nouvel essai d'auscultation des microtunnels en vue de vérifier la qualité des injections de blocage. AFTES, 1999, p. 197-201.

Vanoudheusden E., Petit G., Robert J., Emeriault. F, Kastner R., Lamballerie J.-Y., Reynaud B. - Analysis of movements induced by tunnelling with an earth-pressure balance machine and correlation with excavating parameters. Geotechnical Aspects of Underground Construction in soft Ground, Bakker et al. (eds), Taylor \& Francis Group, London, 2006, p. 81-86. 